\title{
SYNTHESIS, CHARACTERIZATION AND UREASE INHIBITION STUDIES OF TRANSITION METAL COMPLEXES OF THIOUREAS BEARING IBUPROFEN MOIETY
}

\author{
AMARA MUMTAZ ${ }^{a *}$, JAHANZAIB ARSHAD ${ }^{a}$, AAMER SAEED \\ MUHAMMAD AZHAR HYAT NAWAZ AND JAMSHED IQBAL ${ }^{c, d^{*}}$ \\ a Department of Chemistry, COMSATS University, Abbottabad Campus, Pakistan, 22060 \\ ${ }^{b}$ Department of Chemistry, Quaid-i-Azam University, Islamabad, Pakistan 45320 \\ cDepartment of Pharmacy, COMSATS University, Abbottabad Campus, Pakistan, 22060 \\ ${ }^{a}$ Centre for Advanced Drug Research, COMSATS University, Abbottabad Campus, 22060, Pakistan
}

\begin{abstract}
Starting from ibuprofen, a non-steroidal anti-inflammatory drug, $N, N^{\prime}$-disubstituted thiourea derivatives were synthesized by refluxing the acid chloride of ibuprofen with potassium thiocyanate followed by substituted anilines to get $N$-2-(4-(2-methylpropyl)phenyl)propionoyl- $N^{\prime}-\left(2^{\prime}\right.$-bromophenyl) thiourea (1), $N-2$ (4-(2-methylpropyl)phenyl)propionoyl- $N^{\prime}-\left(2^{\prime}\right.$-chlorophenyl) thiourea (2) and $N$-2-(4-(2-methylpropyl)phenyl)propionoyl-N'-(3',4'-dichlorophenyl) thiourea (3). Metal complexes (4-15) of (1-3) were synthesized by refluxing it with one equivalence salts of $\mathrm{Co}$ (II), $\mathrm{Ni}(\mathrm{II}), \mathrm{Pb}(\mathrm{II})$ and $\mathrm{Cu}(\mathrm{I})$. Structures of all the synthesized thiourea ligands and their metal complexes were determined by FTIR and ${ }^{1}$ HNMR spectroscopy. Shift in stretching frequency in FTIR and resonance frequency in ${ }^{1}$ HNMR spectroscopic data suggested that the ligands and metal are coordinated to afford respective metal complexes M[L]. The synthesized compounds were tested against urease enzyme and the results were compared with standard thiourea, as positive control. Most of the investigated compounds showed potential inhibitory activity against Jack bean urease. Compound $\mathbf{5}$ was found to be the most potent urease inhibitor with $\mathrm{IC}_{50}$ of $14.6 \mu \mathrm{M}$, whereas compounds 8 , 11 and 12 possessed potent urease inhibition. The tested compounds can be taken as lead molecules for gastrointestinal ulcer therapy.
\end{abstract}

Key words: Amides, Thioureas, Metal complexes, Ibuprofen, Urease.

\section{INTRODUCTION}

Urease (urea aminohydrolase E.C. 3.5.1.5) is nickel containing metalloenzyme which belongs to the super family of amidohydrolases and phosphotriestreases, responsible for the hydrolytic decomposition of urea (Holm 1997). It was the first enzyme which crystallized from the plant source Canavalia ensiformis and was also the first protein containing nickel in the active site (Saeed et al. 2014; Saeed et al. 2013). Because of these characteristics urease is considered as a paradigm in the development of inorganic biochemistry. Urease catalyses the hydrolysis of urea in different plants, algae, fungi, and microorganisms to produce ammonia and carbamate (Mobley HL 1989). Enzyme catalysed hydrolysis-process occurred at a rate of approximately $10^{14}$ times the rate of the unanalyzed reaction and considered as the final step of nitrogen metabolism in all living organisms. Different types of urease enzymes can be isolated from bacteria, fungi, algae and plants but enzymatic activity is same due to the occarance of the same order of amino acid (Bacanamwo M 2002; Benini S 1999). Urease activity due to infectious microorganism is the major reason for many deceases in humans such as development of infection stones, pyelonephritis, stomach and peptic ulcer, urinary catheter encrustation, and hepatic com (Gripenberg-Lerche C 2000; Mobley et al. 1995; Mobley HL 1989). To overcome these diverse types of problems, urease inhibitors have received special attention over the past few years. These inhibitors generally divided into two classes, substrate structural analogs like hydroxamic acid (Kobashi K 1962,; Krajewska B 2009; Muri EMF 2003) and those which affect the mechanism of the reaction like phosphoramidat (Amtul Z 2002; Kot M 2001). Lansoprazole (Nagata K 1993), omeprazole (Kuehler TC 1995), thiol-compounds (Todd MJ 1989), quinines (Bundy LG 1973) and schiff base derivatives (Hanif M 2012) are reported as potent urease inhibitors.

On the other hand Ibuprofen, an analgesic drug, is famous for its antipyretic and anti-inflammatory properties and found promising to cut the tumor growth in colorectal cancer (KD 2003; McMillan DC 1995; Rao P 2008). The incorporation of ibuprofen moiety in metal complexes of thioureas has opened a new area of research in organometallics (Yuan YF 2001). Thioureas and their derivatives have gained the significance importance in the field of medicinal chemistry because of their biological activities such as: antituberculosis, analgesic, antimicrobial, antiarrhythmic, anti-HIV, anti-inflammatory, rodenticides, fungicide, herbicides and as inhibitors of phenoloxidase enzyme (Kumavat 2013). N, N'-Disubstituted thioureas are of great importance due to their ability to concentrate and separate the toxic metals. Therefore metal complexes of aroyl thiourea are excellent candidates to use as lixiviant, chromogenic ionophore towards fluoride ion detection, in opto-electric modulation, for developing polymer membrane ion-selective electrode and also exhibit great anti-bacterial, anti-fungal, anti-tumor, antiviral, anti-mycobacterial and also posses urease inhibition activities (Arslan et al. 2006; D'Cruz OJ 2003; Del CR 2002; Dhumane NR 2006; Moro AC 2009; Sun MZ 2008; Tremblay L 1996). Thioureas and their derivative are considered as very effective inhibitory active agents against the urease enzyme (Aslam MA 2011). Considerable attention has been focused on transition metal complexes containing thioureas. Thioureas considered as very good chelating agents for transition metal complexes. These complexes play important role to inhibit the over activities of wide range of enzymes due to their specific molecular architectures (Carcu V 2000).

Literature reveals the efficiency of thioureas ligand toward complex formation so the aim of present study is the synthesis of some aroyl thiourea bearing ibuprofen moiety and their metal complexes with $\mathrm{Ni}(\mathrm{II}), \mathrm{Pb}(\mathrm{II}), \mathrm{Co}$ (II) and $\mathrm{Cu}(\mathrm{I})$ and their evaluation as urease inhibitor.

\section{EXPERIMENTAL}

\subsection{Instruments}

Melting points were determined using capillary tubes and an electrothermal melting point apparatus, model MEL-TEMP MP-D Mitamura Riken Kogyo, Japan, and were uncorrected.

Infrared spectra $\left(250-4000 \mathrm{~cm}^{-1}\right)$ were recorded on a Perkin-Elmer system 2000 FT-IR spectrometer and on Perkin-Elmer system 2000 FTIR spectrometer using nujol mull. The ${ }^{1} \mathrm{HNMR}$ were recorded on a Varian Associates Inova spectrometer (400 and $300 \mathrm{MHz}$ ) using deuterated solvents and tetramethyl silane (TMS) as a reference. ${ }^{1} \mathrm{HNMR}$ Spectra were also recorded on Bruker AM-400 NMR spectrophotometer using $\mathrm{CDCl}_{3}$ as an internal reference. Chemical shifts are given in $\delta$ scale (ppm). However the splitting of proton resonances in the ${ }^{1} \mathrm{HNMR}$ spectra is defined as $\mathrm{s}=$ singlet, $\mathrm{d}=$ doublet, $\mathrm{t}=$ triplet and $\mathrm{m}=$ multiplet pattern

2.2 Synthesis of the $N, N$ '-Disubstituted thioureas (1-3)

To a $100 \mathrm{ml}$ of two necked round bottom flask, freshly prepared solution of ibuprofen [2-(4-(2 methylpropyl)phenyl)propanoic acid] $(0.0048 \mathrm{~mol})$ was added to thionyl chloride $(1.5 \mathrm{eq})$ and heated under reflux to give benzoyl chloride. The resulting reaction mixture was added dropwise to a suspension of potassium thiocyanate $(0.0048 \mathrm{~mol})$ and reaction mixture was refluxed for 30 $\mathrm{min}$, then cooled to room temperature. A solution of substituted aniline $(0.0048$ $\mathrm{mol}$ ) in dry acetone was added and the resulting mixture was stirred for $2 \mathrm{~h}$ Completion of reaction was checked by TLC. On cooling, the reaction mixture was slowly poured into acidified $(\mathrm{pH} 4-5)$ chilled water and stirred well with a glass rod. The solid products (1-3) obtained were purified by slow evaporation technique (see Scheme 1) (Rasmussen CR 1988; Rauf MK 2006). 
<smiles>CC(C)Cc1ccc(C(C)C(=O)O)cc1</smiles>

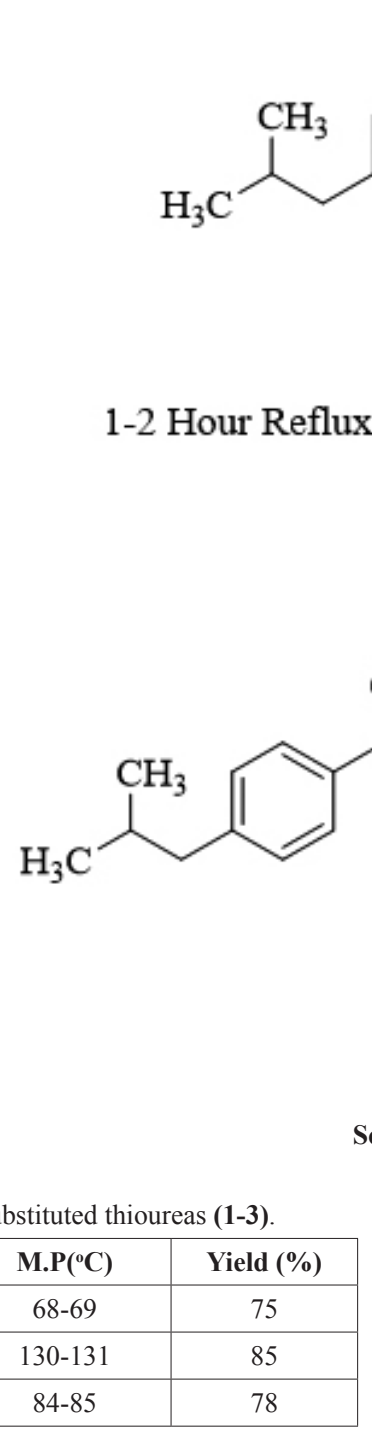

Scheme 1
Table 1. Physical data of the N,N'-disubstituted thioureas (1-3)

\begin{tabular}{|c|c|c|c|}
\hline No. & $\mathbf{R}$ & $\mathbf{M . P}\left({ }^{\circ} \mathbf{C}\right)$ & Yield (\%) \\
\hline $\mathbf{1}$ & $2-\mathrm{Br}$ & $68-69$ & 75 \\
\hline $\mathbf{2}$ & $2-\mathrm{Cl}$ & $130-131$ & 85 \\
\hline $\mathbf{3}$ & 3,4-Dichloro & $84-85$ & 78 \\
\hline
\end{tabular}

$N$-2-(4-(2-methylpropyl)phenyl)propionoyl- $N^{\prime}-\left(2^{\prime}\right.$-bromophenyl) thiourea (1)

Yellow solid, Yield: $75 \%, \mathrm{R}_{\mathrm{f}}(n$-hexane : ethyl acetate, $9: 1)=0.2$. Melting point.: $68-69^{\circ} \mathrm{C}$. IR $\left(\mathrm{cm}^{-1}\right)$ : $v(\mathrm{~N}-\mathrm{H}) 3212(\mathrm{w}), v(\mathrm{C}=\mathrm{O}) 1692(\mathrm{~s}), v(\operatorname{arom} . \mathrm{C}=\mathrm{C})$ $1589(\mathrm{~m}), v(\mathrm{C}=\mathrm{S}) 1236(\mathrm{~s}), v(\mathrm{C}-\mathrm{N}) 1158(\mathrm{~m}) .{ }^{1} \mathrm{HMR}\left(\mathrm{CDCl}_{3}\right): 12.43(\mathrm{~s}, 1 \mathrm{H}$, CSNH), 8.47 (s, 1H, CONH), 7.91 (brdd, 1H, ArH-5' J = 7.8, J = 1.9), 7.59 (dd, $1 \mathrm{H}$, ArH-2', $\mathrm{J}=8.1, \mathrm{~J}=0.9), 7.41$ (dt, 1H, ArH-4', $\mathrm{J}=8.1, \mathrm{~J}=0.9)$ 7.11-7.30 (m, 5H, ArH-2,3,5,6,3') 3.70 (q, 1H, CH-CH $, \mathrm{J}=14.1, \mathrm{~J}=6.9), 2.49$ (d, 2H, Ar- $\left.\mathrm{CH}_{2}-\mathrm{H}, \mathrm{J}=7.2\right), 1.84-1.90\left(\mathrm{~m}, 1 \mathrm{H}, \mathrm{CH}\left(\mathrm{CH}_{3}\right)_{2}\right), 1.58\left(\mathrm{~d}, 3 \mathrm{H}, \mathrm{CH}_{3}-\mathrm{CH}-\mathrm{Ar}, \mathrm{J}\right.$ $\left.=7.2), 0.93\left(\mathrm{~d} 6 \mathrm{H},\left(\mathrm{CH}_{3}\right)_{2}-\mathrm{CH}\right), \mathrm{J}=6.6\right)$.

$N$-2-(4-(2-methylpropyl)phenyl)propionoyl- $N^{\prime}-\left(2^{\prime}\right.$-chlorophenyl) thiourea (2)
Off white solid, Yield: $85 \%, \mathrm{R}_{\mathrm{f}}(n$-hexane : ethyl acetate, $9: 1)=0.4$, Melting point: $130-131^{\circ} \mathrm{C}$, IR $\left(\mathrm{cm}^{-1}\right): v(\mathrm{~N}-\mathrm{H}) 3281(\mathrm{w}), v(\mathrm{C}=\mathrm{O}) 1659$ (s), v (arom. $\mathrm{C}=\mathrm{C}) 1588(\mathrm{~m}), v(\mathrm{C}=\mathrm{S}) 1268(\mathrm{~s}), v(\mathrm{C}-\mathrm{N}) 1176(\mathrm{~m}) .{ }^{1} \mathrm{HMR}\left(\mathrm{CDCl}_{3}\right): 12.39(\mathrm{~s}$, $1 \mathrm{H}, \mathrm{CSNH}), 8.54$ (s, 1H, CONH), 7.09-7.83 (m, 8H, ArH-2,3,5,6,2',3',5',6') $3.73\left(\mathrm{q}, 1 \mathrm{H}, \mathrm{CH}-\mathrm{CH}_{3}, \mathrm{~J}=14.1, \mathrm{~J}=6.9\right), 2.51\left(\mathrm{~d}, 2 \mathrm{H}, \mathrm{Ar}_{-} \mathrm{CH}_{2}-\mathrm{H}, \mathrm{J}=7.2\right)$, 1.87-1.95 (m, 1H, CH- $\left.\left(\mathrm{CH}_{3}\right)_{2}\right), 1.62\left(\mathrm{~d}, 3 \mathrm{H}, \mathrm{CH}_{3}-\mathrm{CH}-\mathrm{Ar}, \mathrm{J}=7.2\right), 0.97$ (d, 6H, $\left.\left.\left(\mathrm{CH}_{3}\right)_{2}-\mathrm{CH}\right), \mathrm{J}=6.6\right)$.

$N$-2-(4-(2-methylpropyl)phenyl)propionoyl- $N^{\prime}-\left(3^{\prime}, 4^{\prime}\right.$-dichloroPhenyl) thiourea (3)

Off white solid. Yield: $78 \% . \mathrm{R}_{\mathrm{f}}(n$-Hexane : Ethyl acetate, 9:1) $=0.3$. Melting point: $84-85^{\circ} \mathrm{C}$, IR $\left(\mathrm{cm}^{-1}\right): v(\mathrm{~N}-\mathrm{H}) 3299(\mathrm{w}), v(\mathrm{C}=\mathrm{O}) 1688(\mathrm{~s}), v$ (arom. $\mathrm{C}=\mathrm{C}) 1575(\mathrm{~m}), v(\mathrm{C}=\mathrm{S}) 1200(\mathrm{~s}), v(\mathrm{C}-\mathrm{N}) 1151(\mathrm{~m}) .{ }^{1} \mathrm{HMR}\left(\mathrm{CDCl}_{3}\right): 12.27(\mathrm{~s}$, 1H, CSNH), 8.69 (s, 1H, CONH), 7.17-7.94 (m, 8H, ArH-2,3,5,6,2',3',5',6') 4.49 (q, 1H, CH-CH $, J=13.8, J=6.9), 2.45\left(\mathrm{~d}, 2 \mathrm{H}, \mathrm{Ar}-\mathrm{CH}_{2}-\mathrm{H}, \mathrm{J}=7.2\right)$, 1.89-1.97 (m, 1H, CH $\left.\left(\mathrm{CH}_{3}\right)_{2}\right), 1.67$ (d, 3H, $\left.\mathrm{CH}_{3}-\mathrm{CH}-\mathrm{Ar}, \mathrm{J}=7.2\right), 0.94$ (d, 6H, $\left.\left.\left(\mathrm{CH}_{3}\right)_{2}-\mathrm{CH}\right), \mathrm{J}=6.6\right)$.

2.3. Synthesis of the Metal Complexes (4-15)

2.3.1. Co(II) complex of $\mathbf{N}, \mathbf{N}^{\prime}$-disubstituted thiourea (4-6)

The Co (II) complexes (4-6) were prepared according to a prescribed method $[9,15]$. The freshly prepared solution of metal salt $\mathrm{CoCl}_{2} \cdot 6 \mathrm{H}_{2} \mathrm{O}$ 
(0.00021 moles) in ethanol (EtOH) was added dropwise to the ligand in a $1: 1$ molar ratio with a small excess of ligand in dichloromethane (DCM) and stirred for 6 hours. The green color precipitates were filtered and recrystallized from an ethanole-dichloromethane mixture (1:1) (Scheme 2) (Douglass 1934; Nadeem S 2009).

Co(II) complex of $\mathbf{N}$-2-(4-(2-methylpropyl)phenyl)propionoyl- $\boldsymbol{N}^{\prime}-\left(2^{\prime}-\right.$ bromophenyl) thiourea (4)

Green solid. Yield: $73 \%$. Melting point: $98-99{ }^{\circ} \mathrm{C}$. IR $\left(\mathrm{cm}^{-1}\right): v(\mathrm{C}=\mathrm{O})$ $1696(\mathrm{~s}), v($ arom. $\mathrm{C}=\mathrm{C}) 1589(\mathrm{~m}), v(\mathrm{C}=\mathrm{S}) 1238(\mathrm{~s}), v(\mathrm{C}-\mathrm{N}) 1162(\mathrm{~m}) .{ }^{1} \mathrm{HMR}$ $\left(\mathrm{CDCl}_{3}\right) 8.36(\mathrm{~s}, 1 \mathrm{H}, \mathrm{CSNH}), 7.01-7.52\left(\mathrm{~m}, 8 \mathrm{H}, \mathrm{ArH}-2,3,5,6,2^{\prime}, 3^{\prime}, 5^{\prime}, 6^{\prime}\right) 3.76$ $\left(\mathrm{q}, 1 \mathrm{H}, \mathrm{CH}-\mathrm{CH}_{3}, \mathrm{~J}=13.8, \mathrm{~J}=6.9\right), 2.38\left(\mathrm{~d}, 2 \mathrm{H}, \mathrm{Ar}-\mathrm{CH}_{2}-\mathrm{H}, \mathrm{J}=7.2\right), 1.84-1.99$ $\left(\mathrm{m}, 1 \mathrm{H}, \mathrm{CH}-\left(\mathrm{CH}_{3}\right)_{2}\right), 1.44\left(\mathrm{~d}, 3 \mathrm{H}, \mathrm{CH}_{3}-\mathrm{CH}-\mathrm{Ar}, \mathrm{J}=7.2\right), 0.86\left(\mathrm{~d}, 6 \mathrm{H},\left(\mathrm{CH}_{3}\right)_{2}-\right.$ $\mathrm{CH}), \mathrm{J}=6.6$ ), Anal, Calc. for $\mathrm{C}_{43} \mathrm{H}_{55} \mathrm{Br}_{2} \mathrm{CoN}_{4} \mathrm{O}_{2} \mathrm{~S}_{2}: \mathrm{C}, 54.78, \mathrm{H}, 5.88, \mathrm{~N}, 5.94$. Found: C, 55.18, H, 5.48, N, 5.54

Co(II) complex of $N$-2-(4-(2-methylpropyl)phenyl)propionoyl- $\boldsymbol{N}^{\prime}-\left(2^{\prime}\right.$ chlorophenyl) thiourea (5)

Green solid. Yield: $79 \%$. Melting point: $95-96{ }^{\circ} \mathrm{C}$. IR $\left(\mathrm{cm}^{-1}\right): v(\mathrm{C}=\mathrm{O})$ $1686(\mathrm{~s}), v($ arom. $\mathrm{C}=\mathrm{C}) 1591(\mathrm{~m}), v(\mathrm{C}=\mathrm{S}) 1231(\mathrm{~s}), v(\mathrm{C}-\mathrm{N}) 1192(\mathrm{~m}) .{ }^{1} \mathrm{HMR}$ $\left(\mathrm{CDCl}_{3}\right) 8.32$ (s, 1H, CSNH), 6.41-6.97 (m, 8H, ArH-2,3,5,6,2',3',5',6') 3.01 (q, 1H, CH-CH $, J=13.8, J=6.9), 1.73\left(\mathrm{~d}, 2 \mathrm{H}, \mathrm{Ar}-\mathrm{CH}_{2}-\mathrm{H}, \mathrm{J}=7.2\right), 1.10-1.26$ $\left(\mathrm{m}, 1 \mathrm{H}, \mathrm{CH}-\left(\mathrm{CH}_{3}\right)_{2}\right), 0.83\left(\mathrm{~d}, 3 \mathrm{H}, \mathrm{CH}_{3}-\mathrm{CH}-\mathrm{Ar}, \mathrm{J}=7.2\right), 0.15\left(\mathrm{~d}, 6 \mathrm{H},\left(\mathrm{CH}_{3}\right)_{2}-\right.$ $\mathrm{CH}), \mathrm{J}=6.6)$, Anal, Calc. for $\mathrm{C}_{43} \mathrm{H}_{55} \mathrm{Cl}_{2} \mathrm{CoN}_{4} \mathrm{O}_{2} \mathrm{~S}_{2}: \mathrm{C}, 60.48, \mathrm{H}, 6.49, \mathrm{~N}, 6.56$ Found: C, 60.88, H, 6.08, N, 6.15.

Co(II) complex of $\mathrm{N}$-2-(4-(2-methylpropyl)phenyl)propionoyl- $N^{\prime}$ $\left(3^{\prime}, 4^{\prime}\right.$-dichlorophenyl) thiourea (6)

Brown solid. Yield: $84 \%$. Melting point: $102-103{ }^{\circ} \mathrm{C}$. IR $\left(\mathrm{cm}^{-1}\right): v(\mathrm{C}=\mathrm{O})$ $1695(\mathrm{~s}), v(\operatorname{arom} . \mathrm{C}=\mathrm{C}) 1576(\mathrm{~m}), v(\mathrm{C}=\mathrm{S}) 1236(\mathrm{~s}), v(\mathrm{C}-\mathrm{N}) 1149(\mathrm{~m}) .{ }^{1} \mathrm{HMR}$ $\left(\mathrm{CDCl}_{3}\right)$ : 7.97 (s, 1H, CSNH), 7.06-7.81 (m, 8H, ArH-2,3,5,6,2',3',5',6') 4.27 $(\mathrm{q}, 1 \mathrm{H}, \mathrm{CH}-\mathrm{CH}, \mathrm{J}=13.8, \mathrm{~J}=6.9), 2.39\left(\mathrm{~d}, 2 \mathrm{H}, \mathrm{Ar}-\mathrm{CH}_{2}-\mathrm{H}, \mathrm{J}=7.2\right), 1.74-1.86$ $\left(\mathrm{m}, 1 \mathrm{H}, \mathrm{CH}-\left(\mathrm{CH}_{3}\right)_{2}\right), 1.52\left(\mathrm{~d}, 3 \mathrm{H}, \mathrm{CH}_{3}-\mathrm{CH}-\mathrm{Ar}, \mathrm{J}=7.2\right), 0.79\left(\mathrm{~d}, 6 \mathrm{H},\left(\mathrm{CH}_{3}\right)_{2}\right.$ $\mathrm{CH}), \mathrm{J}=6.6$ ), Anal, Calc. for $\mathrm{C}_{43} \mathrm{H}_{53} \mathrm{Cl}_{4} \mathrm{CoN}_{4} \mathrm{O}_{2} \mathrm{~S}_{2}: \mathrm{C}, 55.97, \mathrm{H}, 5.79, \mathrm{~N}, 6.07$. Found: C, 55.57, H, 6.19, N, 6.03.

2.3.2. $\mathrm{Ni}(\mathrm{II})$ complex of $N, N^{\prime}$-disubstituted thiourea (7-9)

A solution of the $\mathrm{Ni}\left(\mathrm{CH}_{3} \mathrm{COO}\right)(0.00021 \mathrm{~mol})$ in $\mathrm{EtOH}(4 \mathrm{ml})$ was added dropwise to a solution of the ligand $(0.00042 \mathrm{~mol})$ in DCM $(13 \mathrm{ml})$ at room temperature and the resulting reaction mixture was stirred for 4 hours. The solid products obtained were filtered and recrystallized from dichloromethaneethanol mixture (1:1) (Scheme 3) (Beyer L 1975; Douglass 1934).

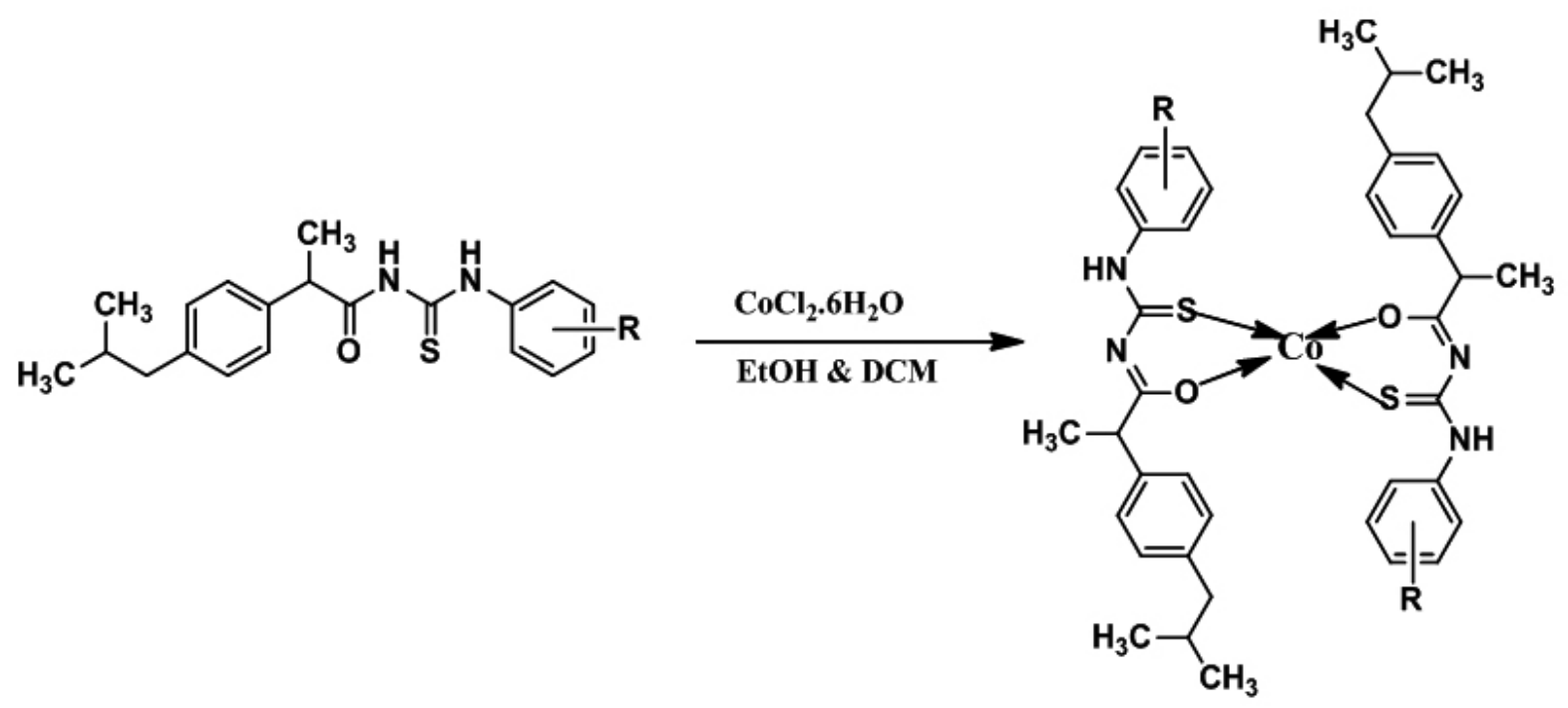

Scheme 2<smiles>[R]c1ccc(NC(=S)NC(=O)C(C)c2ccc(CC(C)C)cc2)cc1</smiles>

\section{$\mathrm{Ni}\left(\mathrm{CH}_{3} \mathrm{COO}\right)_{2} \cdot \mathbf{4} \mathrm{H}_{2} \mathrm{O}$ EtOH \& DCM}

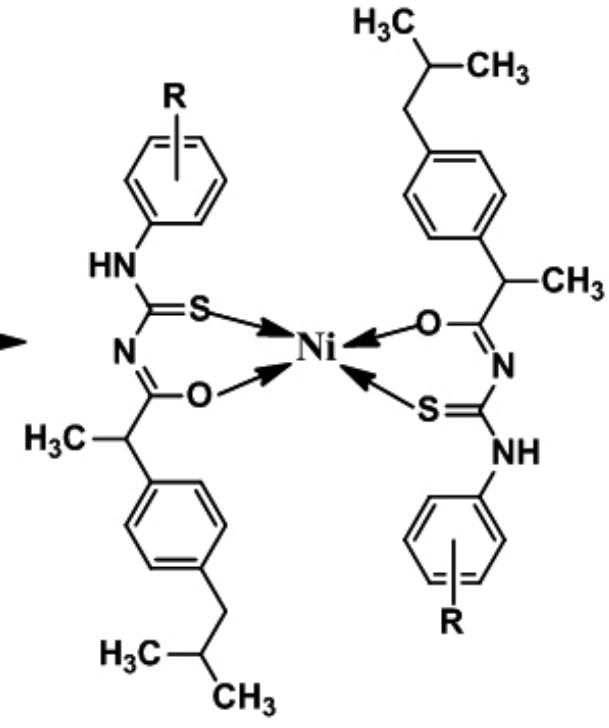

Scheme 3 
Ni(II) complex of $N$-2-(4-(2-methylpropyl)phenyl)propionoyl- $N^{\prime}-\left(2^{\prime}\right.$ bromophenyl) thiourea (7)

Yellow solid. Yield: 59\%. Melting point: $91-92{ }^{\circ} \mathrm{C}$. IR $\left(\mathrm{cm}^{-1}\right): v(\mathrm{C}=\mathrm{O})$ $1659(\mathrm{~s}), v(\operatorname{arom} . \mathrm{C}=\mathrm{C}) 1587(\mathrm{~m}), v(\mathrm{C}=\mathrm{S}) 1241(\mathrm{~s}), v(\mathrm{C}-\mathrm{N}) 1148(\mathrm{~m}) .{ }^{1} \mathrm{HMR}$ $\left(\mathrm{CDCl}_{3}\right) 8.29$ (s, 1H, CSNH), 7.06-7.59 (m, 8H, ArH-2,3,5,6,2',3',5',6') 3.81 $\left(\mathrm{q}, 1 \mathrm{H}, \mathrm{CH}-\mathrm{CH}_{3}, \mathrm{~J}=13.8, \mathrm{~J}=6.9\right), 2.40\left(\mathrm{~d}, 2 \mathrm{H}, \mathrm{Ar}-\mathrm{CH}_{2}-\mathrm{H}, \mathrm{J}=7.2\right), 1.72-1.87$ $\left(\mathrm{m}, 1 \mathrm{H}, \mathrm{CH}-\left(\mathrm{CH}_{3}\right)_{2}\right), 1.38$ (d, 3H, CH $\left.-\mathrm{CH}-\mathrm{Ar}, \mathrm{J}=7.2\right), 0.85\left(\mathrm{~d}, 6 \mathrm{H},\left(\mathrm{CH}_{3}\right)-\right.$ $\mathrm{CH}), \mathrm{J}=6.6$ ), Anal, Calc. for $\mathrm{C}_{43} \mathrm{H}_{53} \mathrm{Cl}_{4} \mathrm{NiN}_{4} \mathrm{O}_{2} \mathrm{~S}_{2}: \mathrm{C}, 55.97, \mathrm{H}, 5.79, \mathrm{~N}, 6.07$. Found: C, 55.57, H, 6.19, N, 6.03.

$\mathrm{Ni}$ (II) complex of $\boldsymbol{N}$-2-(4-(2-methylpropyl)phenyl)propionoyl- $\boldsymbol{N}^{\prime}-\left(2^{\prime}-\right.$ chlorophenyl) thiourea (8)

Yellowish orange. Yield: $67 \%$. Melting point: $100-101^{\circ} \mathrm{C}$. IR $\left(\mathrm{cm}^{-1}\right): v$ $(\mathrm{C}=\mathrm{O}) 1691(\mathrm{~s}), v(\operatorname{arom} . \mathrm{C}=\mathrm{C}) 1589(\mathrm{~m}), v(\mathrm{C}=\mathrm{S}) 1250(\mathrm{~s}), v(\mathrm{C}-\mathrm{N}) 1158(\mathrm{~m})$. ${ }^{1} \mathrm{HMR}\left(\mathrm{CDCl}_{3}\right) 8.18$ (s, 1H, CSNH), 6.87-7.39 (m, 8H, ArH-2,3,5,6,2',3',5',6') $3.51\left(\mathrm{q}, 1 \mathrm{H}, \mathrm{CH}-\mathrm{CH}_{3}, \mathrm{~J}=13.8, \mathrm{~J}=6.9\right), 2.42\left(\mathrm{~d}, 2 \mathrm{H}, \mathrm{Ar}-\mathrm{CH}_{2}-\mathrm{H}, \mathrm{J}=7.2\right)$, 1.56-1.69 (m, 1H, CH- $\left.\left(\mathrm{CH}_{3}\right)_{2}\right), 1.36$ (d, 3H, $\left.\mathrm{CH}_{3}-\mathrm{CH}-\mathrm{Ar}, \mathrm{J}=7.2\right), 0.62(\mathrm{~d}, 6 \mathrm{H}$, $\left.\left.\left(\mathrm{CH}_{3}\right)_{2}-\mathrm{CH}\right), \mathrm{J}=6.6\right)$, Anal, Calc. for $\mathrm{C}_{43} \mathrm{H}_{55} \mathrm{Br}_{2} \mathrm{~N}_{4} \mathrm{NiO}_{2} \mathrm{~S}_{2}: \mathrm{C}, 54.79, \mathrm{H}, 5.88, \mathrm{~N}$, 5.94. Found: C, 54.39, H, 5.48, N, $6.34 \%$

$\mathrm{Ni}$ (II) complex of $\mathrm{N}$-2-(4-(2-methylpropyl)phenyl)propionoyl- $\mathrm{N}^{\prime}$ $\left(3^{\prime}, 4^{\prime}\right.$-dichlorophenyl) thiourea (9)
Color: brown. Yield: $84 \%$ m.p. $102-103{ }^{\circ} \mathrm{C} \mathrm{dec}$. IR $\left(\mathrm{cm}^{-1}\right): v(\mathrm{~N}-\mathrm{H}) 3387$ $(\mathrm{w}), v(\mathrm{C}=\mathrm{O}) 1692(\mathrm{~s}), v(\operatorname{arom} . \mathrm{C}=\mathrm{C}) 1576(\mathrm{~m}), v(\mathrm{C}=\mathrm{S}) 1236(\mathrm{~s}), v(\mathrm{C}-\mathrm{N})$ 1149 (m), NMR data; ${ }^{1} \mathrm{HMR}\left(\mathrm{CDCl}_{3}\right) 7.97$ (s, 1H, CSNH), 7.06-7.81 (m, 8H, $\left.\mathrm{ArH}-2,3,5,6,2^{\prime}, 3^{\prime}, 5^{\prime}, 6^{\prime}\right) 4.27$ (q, 1H, CH-CH, J = 13.8, J = 6.9), 2.39 (d, 2H, Ar- $\left.\mathrm{CH}_{2}-\mathrm{H}, \mathrm{J}=7.2\right), 1.74-1.86\left(\mathrm{~m}, 1 \mathrm{H}, \mathrm{CH}-\left(\mathrm{CH}_{3}\right)_{2}\right), 1.52$ (d, 3H, $\mathrm{CH}_{3}-\mathrm{CH}-\mathrm{Ar}, \mathrm{J}$ $\left.=7.2), 0.79\left(\mathrm{~d}, 6 \mathrm{H},\left(\mathrm{CH}_{3}\right)_{2}-\mathrm{CH}\right), \mathrm{J}=6.6\right)$. Anal, Calc. for $\mathrm{C}_{43} \mathrm{H}_{55} \mathrm{Cl}_{4} \mathrm{~N}_{4} \mathrm{NiO}_{2} \mathrm{~S}_{2}$ : C, 55.98, H, 5.79, N, 6.07. Found: C, 56.38, H, 5.39, N, 6.03.

2.3.3. $\mathbf{P b}(\mathrm{II})$ complex of $\mathbf{N}, \mathbf{N}^{\prime}$-disubstituted thiourea (10-12)

Synthesis of $\mathrm{K}_{2}\left[\mathrm{PbCl}_{4}\right]$

$\mathrm{K}\left[\mathrm{PbCl}_{4}\right]$ was synthesized by reacting excess of $\mathrm{KCl}$ with $\mathrm{PbCl}_{2}$. In this regard $\mathrm{PbCl}_{2}(0.000179 \mathrm{~mol})$ was suspended in methanol and $\mathrm{KCl}$ solution was added drop-wise to above solution and reaction mixture was stirred for 3 hours. At the end, the off white suspension obtained was cooled in ice bath for 1 hour to afford off white precipitates of $\mathrm{K}_{2}\left[\mathrm{PbCl}_{4}\right]$.

For the synthesis of $\mathrm{Pb}(\mathrm{II})$ complexes (12-15) the ligand solution $(0.000358$ moles) in $10 \mathrm{~mL}$ of methanol was added drop wise to above solution of $\mathrm{K}_{2}\left[\mathrm{PbCl}_{4}\right]$ and the reaction mixture was stirred for 6 hours. The yellowish precipitates were filtered off and allowed to stand at room temperature for 2 days. Purification of the crude was carried out by using methanol as recrystalising solvent (Scheme 4) (Rauf MK 2010)

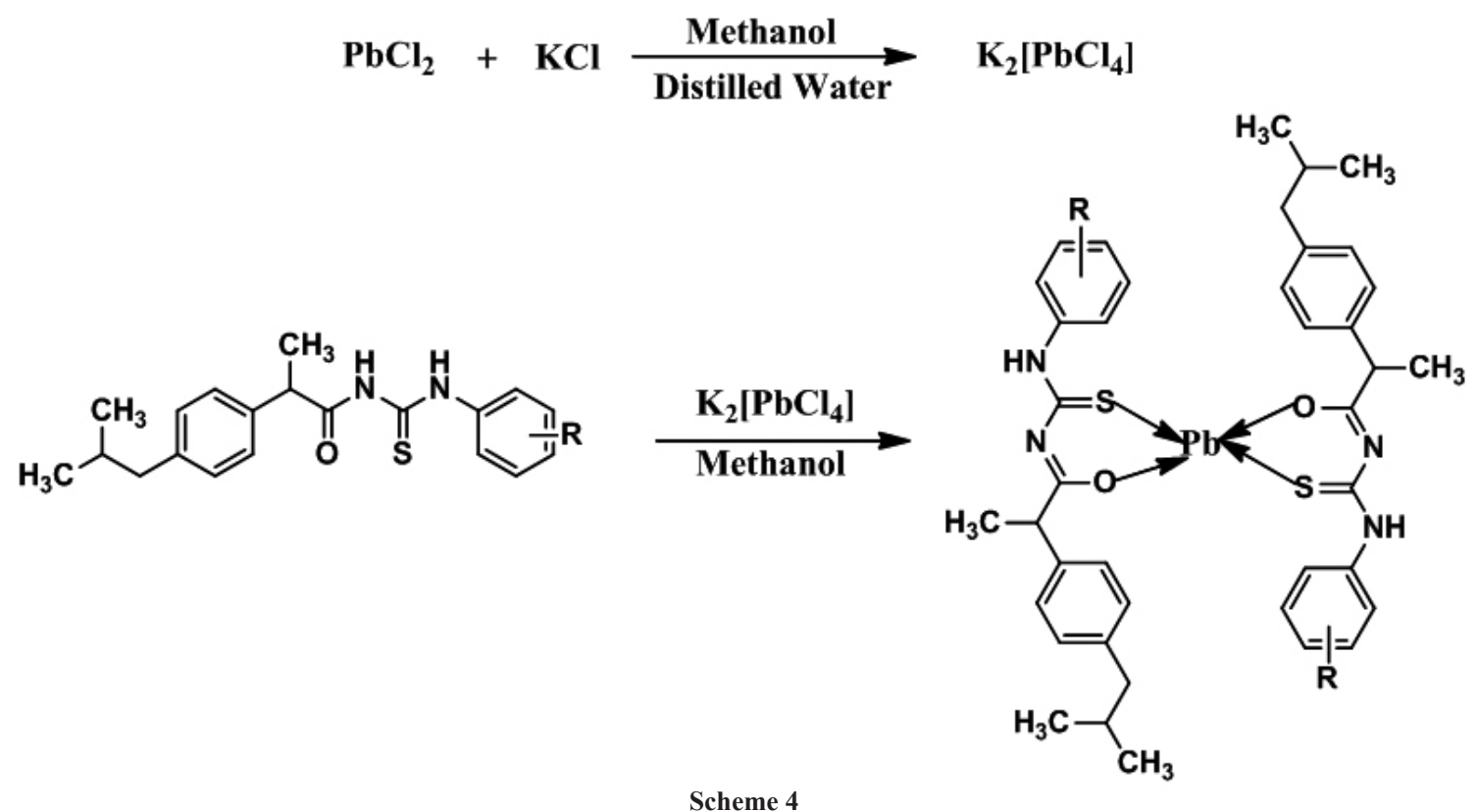

$\mathrm{Pb}$ (II) complex of $\mathrm{N}-2-(4-(2-m e t h y l p r o p y l) p h e n y l) p r o p i o n o y l-N^{\prime}-(2 '-$ bromophenyl) thiourea (10)

Yellow solid. Yield: $78 \%$. Melting point. $90-91^{\circ} \mathrm{C}$. IR $\left(\mathrm{cm}^{-1}\right): v(\mathrm{C}=\mathrm{O})$ $1667(\mathrm{~s}), v(\operatorname{arom} . \mathrm{C}=\mathrm{C}) 1589(\mathrm{~m}), v(\mathrm{C}=\mathrm{S}) 1239(\mathrm{~s}), v(\mathrm{C}-\mathrm{N}) 1159(\mathrm{~m}) .{ }^{1} \mathrm{HMR}$ $\left(\mathrm{CDCl}_{3}\right) 8.19(\mathrm{~s}, 1 \mathrm{H}, \mathrm{CSNH}) 7.47(\mathrm{dd}, 1 \mathrm{H}, \mathrm{ArH}-5, \mathrm{~J}=7.8, \mathrm{~J}=1.5), 7.37$ (dd, $1 \mathrm{H}, \mathrm{ArH}-2^{\prime}, \mathrm{J}=8.1, \mathrm{~J}=1.5$ ), 7.30 (d, 1H, ArH-4', J = 7.8) 7.19-7.27 (m, 4H, ArH-2,3,5,6) 7.14 (d, 1H, ArH-3', J = 8.1) $3.88\left(\mathrm{q}, 1 \mathrm{H}, \mathrm{CH}-\mathrm{CH}_{3}, \mathrm{~J}=13.8, \mathrm{~J}\right.$ $=6.9), 2.46\left(\mathrm{~d}, 2 \mathrm{H}, \mathrm{Ar}-\mathrm{CH}_{2}-\mathrm{H}, \mathrm{J}=7.2\right), 1.78-1.91\left(\mathrm{~m}, 1 \mathrm{H}, \mathrm{CH}-\left(\mathrm{CH}_{3}\right)_{2}\right), 1.50$ (d, 3H, CH -CH-Ar, J = 7.2), $0.89(\mathrm{~d} 6 \mathrm{H},(\mathrm{CH})-\mathrm{CH}), \mathrm{J}=6.6)$, Anal, Calc. for $\mathrm{C}_{43} \mathrm{H}_{55} \mathrm{Br}_{2} \mathrm{~N}_{4} \mathrm{O}_{2} \mathrm{PdS}_{2}$ : C, 52.15, H, 5.60, N, 5.66. Found: C, 51.85, H, 5.20, N, $6.06 \%$.

$\mathrm{Pb}$ (II) complex of $\mathrm{N}-2-(4-(2-m e t h y l p r o p y l) p h e n y l) p r o p i o n o y l-N^{\prime}-(2 '-$ chlorophenyl) thiourea (11)

Yellow solid. Yield: $91 \%$. Melting point. $118-119^{\circ} \mathrm{C}$. IR $\left(\mathrm{cm}^{-1}\right): v(\mathrm{C}=\mathrm{O})$ $1681(\mathrm{~s}), v(\operatorname{arom} . \mathrm{C}=\mathrm{C}) 1589(\mathrm{~m}), v(\mathrm{C}=\mathrm{S}) 1250(\mathrm{~s}), v(\mathrm{C}-\mathrm{N}) 1158(\mathrm{~m}) .{ }^{1} \mathrm{HMR}$ $\left(\mathrm{CDCl}_{3}\right) 8.26(\mathrm{~s}, 1 \mathrm{H}, \mathrm{CSNH}), 6.91-7.46\left(\mathrm{~m}, 8 \mathrm{H}, \mathrm{ArH}-2,3,5,6,2^{\prime}, 3^{\prime}, 5^{\prime}, 6^{\prime}\right) 3.29$ $\left(\mathrm{q}, 1 \mathrm{H}, \mathrm{CH}-\mathrm{CH}_{3}, \mathrm{~J}=14.1, \mathrm{~J}=6.9\right), 2.31\left(\mathrm{~d}, 2 \mathrm{H}, \mathrm{Ar}-\mathrm{CH}_{2}-\mathrm{H}, \mathrm{J}=7.2\right), 1.63-1.79$ $\left(\mathrm{m}, 1 \mathrm{H}, \mathrm{CH}-\left(\mathrm{CH}_{3}\right)_{2}\right), 1.41\left(\mathrm{~d}, 3 \mathrm{H}, \mathrm{CH}_{3}-\mathrm{CH}-\mathrm{Ar}, \mathrm{J}=7.2\right), 0.71\left(\mathrm{~d}, 6 \mathrm{H},\left(\mathrm{CH}_{3}\right)_{2}-\right.$ $\mathrm{CH}), \mathrm{J}=6.6$ ), Anal, Calc. for $\mathrm{C}_{43} \mathrm{H}_{55} \mathrm{Cl}_{2} \mathrm{~N}_{4} \mathrm{O}_{2} \mathrm{PdS}_{2}: \mathrm{C}, 57.30, \mathrm{H}, 6.15, \mathrm{~N}, 6.22$. Found: C, 57.70, H, 6.55, N, $6.44 \%$.

$\mathrm{Pb}$ (II) complex of N-2-(4-(2-methylpropyl)phenyl)propionoyl-N'$\left(3^{\prime}, 4^{\prime}\right.$-dichlorophenyl) thiourea (12)

Yellow solid. Yield: $71 \%$. Melting point $105-106^{\circ} \mathrm{C}$. IR $\left(\mathrm{cm}^{-1}\right): v(\mathrm{C}=\mathrm{O})$ $1692(\mathrm{~s}), v(\operatorname{arom} . \mathrm{C}=\mathrm{C}) 1576(\mathrm{~m}), v(\mathrm{C}=\mathrm{S}) 1265(\mathrm{~s}), v(\mathrm{C}-\mathrm{N}) 1150(\mathrm{~m}) .{ }^{1} \mathrm{HMR}$ $\left(\mathrm{CDCl}_{3}\right) 7.72(\mathrm{~s}, 1 \mathrm{H}, \mathrm{CSNH}), 7.16-7.64\left(\mathrm{~m}, 8 \mathrm{H}, \mathrm{ArH}-2,3,5,6,2^{\prime}, 3^{\prime}, 5^{\prime}, 6^{\prime}\right) 4.28$ $(\mathrm{q}, 1 \mathrm{H}, \mathrm{CH}-\mathrm{CH}, \mathrm{J}=14.1, \mathrm{~J}=6.9), 2.44\left(\mathrm{~d}, 2 \mathrm{H}, \mathrm{Ar}-\mathrm{CH}_{2}-\mathrm{H}, \mathrm{J}=7.2\right), 1.82-1.98$ $\left(\mathrm{m}, 1 \mathrm{H}, \mathrm{CH}-\left(\mathrm{CH}_{3}\right)_{2}\right), 1.59$ (d, 3H, $\left.\mathrm{CH}_{3}-\mathrm{CH}-\mathrm{Ar}, \mathrm{J}=7.2\right), 0.91\left(\mathrm{~d}, 6 \mathrm{H},\left(\mathrm{CH}_{3}\right)_{2}-\right.$ $\mathrm{CH}), \mathrm{J}=6.6$ ), Anal, Calc. for $\mathrm{C}_{43} \mathrm{H}_{53} \mathrm{Cl}_{4} \mathrm{~N}_{4} \mathrm{O}_{2} \mathrm{PdS}_{2}: \mathrm{C}, 53.23, \mathrm{H}, 5.51, \mathrm{~N}, 5.77$. Found: C, 543.63, H, 5.11, N, $6.17 \%$

\subsection{4. $\mathrm{Cu}(\mathrm{I})$ complex of $\mathrm{N}, \mathrm{N}^{\prime}$-Disubstituted thiourea (13-15)}

For the synthesis of $\mathrm{Cu}(\mathrm{I})$ complexes (13-15) thiourea ligand $(0.000625$ mol) was dissolved in $35 \mathrm{ml}$ of acidified methanol $(3.5 \mathrm{ml}, 0.5 \% \mathrm{HCl})$ on stirring. Powdered $\mathrm{Cu}(\mathrm{I}) \mathrm{Cl}(0.00025$ moles $)$ was dissolved in the above solution and resulting suspension was allowed to stir for 4 hours. The yellowish precipitates of $\mathrm{Cu}(\mathrm{I})$ complexes were filtered and washed well with cold methanol.The solid products were purified by slow evaporation technique (Scheme 5) (Rauf MK 2009).

$\mathrm{Cu}(\mathrm{I})$ complex of N-2-(4-(2-methylpropyl)phenyl)propionoyl-N'-(2'bromophenyl) thiourea (13)

Yellow solid. Yield: $74 \%$. Melting point. $82-83{ }^{\circ} \mathrm{C}$. IR $\left(\mathrm{cm}^{-1}\right): v(\mathrm{C}=\mathrm{O})$ $1666(\mathrm{~s}), v(\operatorname{arom} . \mathrm{C}=\mathrm{C}) 1585(\mathrm{~m}), v(\mathrm{C}=\mathrm{S}) 1238(\mathrm{~s}), v(\mathrm{C}-\mathrm{N}) 1168(\mathrm{~m}) .{ }^{1} \mathrm{HMR}$ $\left(\mathrm{CDCl}_{3}\right) 8.45(\mathrm{~s}, 1 \mathrm{H}, \mathrm{CSNH}) 7.02-7.93\left(\mathrm{~m}, 8 \mathrm{H}, \mathrm{ArH}-2,3,5,6,2^{\prime}, 3^{\prime}, 4^{\prime}, 5^{\prime}\right) 3.82$ $\left(\mathrm{q}, 1 \mathrm{H}, \mathrm{CH}-\mathrm{CH}_{3}, \mathrm{~J}=13.8, \mathrm{~J}=6.9\right), 2.41\left(\mathrm{~d}, 2 \mathrm{H}, \mathrm{ArCH}_{2}-\mathrm{H}, \mathrm{J}=7.2\right), 1.81-1.94$ $\left(\mathrm{m}, 1 \mathrm{H}, \mathrm{CH}-\left(\mathrm{CH}_{3}\right)\right), 1.64\left(\mathrm{~d}, 3 \mathrm{H}, \mathrm{CH}_{3}-\mathrm{CH}-\mathrm{Ar}, \mathrm{J}=7.2\right), 0.84\left(\mathrm{~d}, 6 \mathrm{H},\left(\mathrm{CH}_{3}\right)_{2}-\right.$ $\mathrm{CH}), \mathrm{J}=6.6$ ), Anal, Calc. for $\mathrm{C}_{43} \mathrm{H}_{55} \mathrm{Br}_{2} \mathrm{CuN}_{4} \mathrm{O}_{2} \mathrm{~S}_{2}: \mathrm{C}, 54.15, \mathrm{H}, 5.85, \mathrm{~N}, 5.91$. Found: C, 54.55, H, 5.45, N, 6.31\%.

$\mathrm{Cu}(\mathrm{I})$ complex of $\mathrm{N}-2-(4-(2-m e t h y l p r o p y l) p h e n y l)$ propionoyl-N'-(2'chlorophenyl) thiourea (14)

Yellow solid. Yield: $54 \%$. Melting point. $110-111^{\circ} \mathrm{C}$. IR $\left(\mathrm{cm}^{-1}\right)$ : IR $\left(\mathrm{cm}^{-1}\right)$ : 
$v(\mathrm{C}=\mathrm{O}) 1692(\mathrm{~s}), v(\operatorname{arom} . \mathrm{C}=\mathrm{C}) 1588(\mathrm{~m}), v(\mathrm{C}=\mathrm{S}) 1261(\mathrm{~s}), v(\mathrm{C}-\mathrm{N}) 1175(\mathrm{~m})$ ${ }^{1} \mathrm{HMR}\left(\mathrm{CDCl}_{3}\right) 8.39$ (s, 1H, CSNH) 6.97-7.59 (m, 8H, ArH-2,3,5,6,2',3',4',5') $3.44\left(\mathrm{q}, 1 \mathrm{H}, \mathrm{CH}-\mathrm{CH}_{3}, \mathrm{~J}=13.8, \mathrm{~J}=6.9\right), 2.26\left(\mathrm{~d}, 2 \mathrm{H}, \mathrm{Ar}-\mathrm{CH}_{2}-\mathrm{H}, \mathrm{J}=7.2\right)$, 1.55-1.72 (m, 1H, CH-(CH $)$ ), $1.29\left(\mathrm{~d}, 3 \mathrm{H}, \mathrm{CH}_{3}-\mathrm{CH}-\mathrm{Ar}, \mathrm{J}=7.2\right), 0.68(\mathrm{~d}, 6 \mathrm{H}$ $\left.\left(\mathrm{CH}_{3}\right)_{2}-\mathrm{CH}\right), \mathrm{J}=6.6$ ), Anal, Calc. for $\mathrm{C}_{43} \mathrm{H}_{55} \mathrm{Cl}_{2} \mathrm{CuN}_{4} \mathrm{O}_{2} \mathrm{~S}_{2}: \mathrm{C}, 60.16, \mathrm{H}, 6.46, \mathrm{~N}$, 6.53. Found: C, $60.46, \mathrm{H}, 6.06, \mathrm{~N}, 6.93 \%$.

$\mathrm{Cu}(\mathrm{I})$ complex of $\mathrm{N}$-2-(4-(2-methylpropyl)phenyl)propionoyl-N'$\left(3^{\prime}, 4^{\prime}\right.$-dichlorophenyl) thiourea (15)
Yellow solid. Yield: $81 \%$. Melting point. $149-150^{\circ} \mathrm{C}$. IR $\left(\mathrm{cm}^{-1}\right): v(\mathrm{C}=\mathrm{O})$ $1693(\mathrm{~s}), v(\operatorname{arom} . \mathrm{C}=\mathrm{C}) 1505(\mathrm{~m}), v(\mathrm{C}=\mathrm{S}) 1268(\mathrm{~s}), v(\mathrm{C}-\mathrm{N}) 1151(\mathrm{~m}) .{ }^{1} \mathrm{HMR}$ $\left(\mathrm{CDCl}_{3}\right) 7.89$ (s, 1H, CSNH), 7.11-7.77 (m, 8H, ArH-2,3,5,6,2',3',5',6') 4.31 $(\mathrm{q}, 1 \mathrm{H}, \mathrm{CH}-\mathrm{CH}, \mathrm{J}=13.8, \mathrm{~J}=6.9), 2.53\left(\mathrm{~d}, 2 \mathrm{H}, \mathrm{Ar}-\mathrm{CH}_{2}-\mathrm{H}, \mathrm{J}=7.2\right), 1.81-1.92$ $\left(\mathrm{m}, 1 \mathrm{H}, \mathrm{CH}-\left(\mathrm{CH}_{3}\right)_{2}\right), 1.58\left(\mathrm{~d}, 3 \mathrm{H}, \mathrm{CH}_{3}-\mathrm{CH}-\mathrm{Ar}, \mathrm{J}=7.2\right), 0.96\left(\mathrm{~d}, 6 \mathrm{H},\left(\mathrm{CH}_{3}\right)_{2}\right.$ $\mathrm{CH}), \mathrm{J}=6.6)$, Anal, Calc. for $\mathrm{C}_{43} \mathrm{H}_{53} \mathrm{Br}_{2} \mathrm{CuN}_{4} \mathrm{O}_{2} \mathrm{~S}_{2}$ : C, 55.69, H, 5.76, N, 6.04. Found: C, 55.29, H, 5.39, N, $6.44 \%$<smiles>[R]c1ccc(NC(=S)NC(=O)C(C)c2ccc(CC(C)C)cc2)cc1</smiles>

Scheme 5

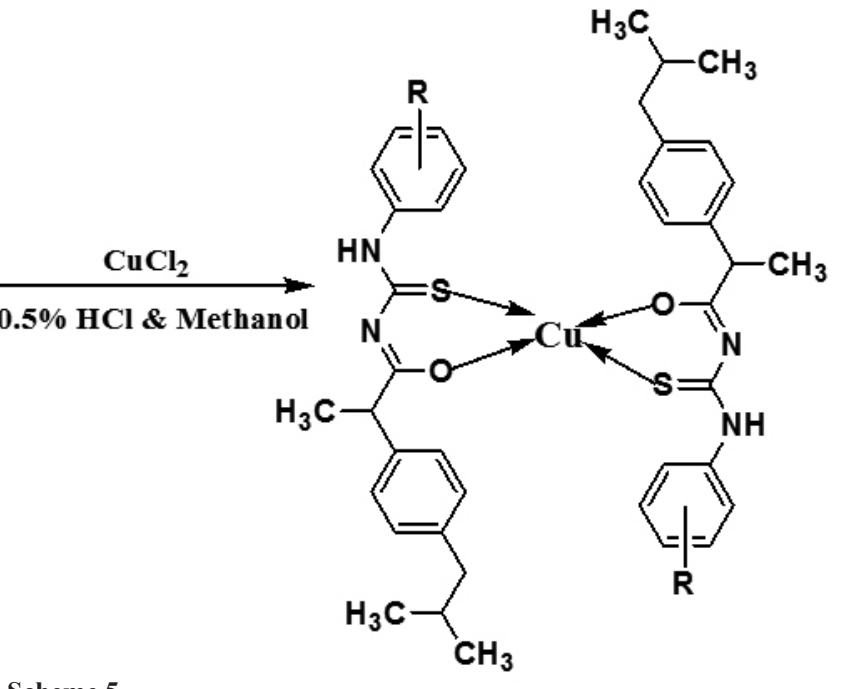

Table 2. Physical Data of the metal complexes of N,N'-disubstituted thioureas (4-15).

\begin{tabular}{|c|c|c|c|c|}
\hline No. & $\mathbf{R}$ & $\mathbf{M X}$ & $\mathbf{M . P}\left({ }^{\circ} \mathbf{C}\right)$ & Yield (\%) \\
\hline $\mathbf{4}$ & $2-\mathrm{Br}$ & $\mathrm{Co}$ & $98-99$ & 73 \\
\hline $\mathbf{5}$ & $2-\mathrm{Cl}$ & $\mathrm{Co}$ & $95-96$ & 79 \\
\hline $\mathbf{6}$ & $3,4-\mathrm{Dichloro}$ & $\mathrm{Co}$ & $102-103$ & 84 \\
\hline $\mathbf{7}$ & $2-\mathrm{Br}$ & $\mathrm{Ni}$ & $91-92$ & 59 \\
\hline $\mathbf{8}$ & $2-\mathrm{Cl}$ & $\mathrm{Ni}$ & $100-101$ & 67 \\
\hline $\mathbf{9}$ & $3,4-\mathrm{Dichloro}$ & $\mathrm{Ni}$ & $102-103$ & 84 \\
\hline $\mathbf{1 0}$ & $2-\mathrm{Br}$ & $\mathrm{Pb}$ & $90-91$ & 98 \\
\hline $\mathbf{1 1}$ & $2-\mathrm{Cl}$ & $\mathrm{Pb}$ & $118-119$ & 71 \\
\hline $\mathbf{1 2}$ & $3,4-\mathrm{Dichloro}$ & $\mathrm{Pb}$ & $105-106$ & 91 \\
\hline $\mathbf{1 3}$ & $2-\mathrm{Br}$ & $\mathrm{Cu}$ & $82-83$ & 74 \\
\hline $\mathbf{1 4}$ & $2-\mathrm{Cl}$ & $\mathrm{Cu}$ & $110-111$ & 54 \\
\hline $\mathbf{1 5}$ & $3,4-\mathrm{Dichloro}$ & $\mathrm{Cu}$ & $149-150$ & 81 \\
\hline
\end{tabular}

\subsection{Urease inhibition Assay}

The amount of ammonia produced was measured by indophenol method and its absorbance provided the enzyme activity (Weatherburn 1967). The assay mixture containing $40 \mu \mathrm{L}$ buffer $(100 \mathrm{mM}$ urea, $1 \mathrm{mM}$ EDTA, 0.01 $\left.\mathrm{M} \mathrm{K}_{2} \mathrm{HPO}_{4}, 0.01 \mathrm{M} \mathrm{LiCl}_{2}, \mathrm{pH} 8.2\right), 10 \mu \mathrm{L}$ of test compound and $10 \mu \mathrm{L}$ of enzyme $(5 \mathrm{U} / \mathrm{mL})$ were incubated in a 96 well plate for 30 minutes at $37^{\circ} \mathrm{C}$. In addition, solutions of $40 \mu \mathrm{L}$ of phenol reagent $(1 \%$, w $/ v$ phenol, $0.005 \%$, w/v sodium nitroprusside) and $40 \mu \mathrm{L}$ of alkali reagent $(0.5 \%$, w/v $\mathrm{NaOH}, 0.1 \%$ active chloride $\mathrm{NaOCl}$ ) were added to each well. Experiments were performed in triplicate and thiourea was used as reference drug. Microplate reader (BioTekELx 800, Instruments, Inc., USA) was used to read the absorbance at 625 nm (Kumavat PP 2013).

\section{RESULTS AND DISCUSSIONS}

\subsection{Infrared spectra}

The FTIR spectral data of $\mathrm{N} \mathrm{N}^{\prime}$-disubstituted thioureas (1-3) show absorption bands at $v_{\max } / \mathrm{cm}^{-1} 3281-3381,3442-3462(\mathrm{NH}), 1659-1692(\mathrm{C}=\mathrm{O})$, $1527-1595(\mathrm{CN}), \quad 1575-1589 \quad(\mathrm{C}=\mathrm{C}), \quad 1200-1236 \quad(\mathrm{C}=\mathrm{S}), 1148-1162(\mathrm{C}-\mathrm{S})$ 1151-1171 (CN). The addition of the amine to the $\mathrm{C}=\mathrm{N}$ double bond results in the formation of the desired compounds. As a consequence, the strong band at around $2000 \mathrm{~cm}^{-1}(\mathrm{~N}=\mathrm{C}=\mathrm{S})$ in the isocyanate disappears. Instead of the having normal carbonyl absorption around $v_{\max } / \mathrm{cm}^{-1} 1710$ and a medium $(\mathrm{C}=\mathrm{O})$ absorption band around 1659-1692 indicated a possible hydrogen bond formation between the $\mathrm{H}$-atom of the $\mathrm{CSNH}$ and the $\mathrm{O}$-atom of the carbonyl group (Scheme 1). A comparative absorption pattern study of the complexes (4-15) with the values of the free ligand suggested a significant effect in stretching frequencies of $(\mathrm{CN})$ and $(\mathrm{CS})$ groups due to coordination of the ligand to the $\mathrm{Co}, \mathrm{Ni}, \mathrm{Pb}$ and $\mathrm{Cu}$ atom. In the FTIR spectra of the $\mathrm{N}, \mathrm{N}^{\prime}-$ disubstituted thiourea ligands (1-3) the N-H stretching vibration is observed in the range $3212-3299 \mathrm{~cm}^{-1}$ as a broad band. This band disappears upon metal complex formation of related ligands. This behavior suggests that the ligand is coordinated to the metal atom and lost amine proton. Moreover, the slight shift in stretching frequency of $\mathrm{C}=\mathrm{O}$ in coordinated compound as compared to ligand also accounted the involvement of the carbonyl group in coordination which confirmed the formation of desire compound(Hakan A 2003; Losada $\mathrm{J}$ 2000). A shift in frequency was also be expected for the $\mathrm{C}=\mathrm{S}$ stretching vibration but this stretching vibration could not be assigned unambiguously, because it is located in the fingerprint zone of the IR spectra (Costa 1984).

$3.2{ }^{l} H N M R$ spectra

In the ${ }^{1} \mathrm{HNMR}$ spectral data of ligands (1-3) show that the NH hydrogen resonates at a frequency significantly downfield from other resonances in the spectra. The proton chemical shifts of both $\mathrm{CONH}$ and $\mathrm{CSNH}$ are found around 12.27-12.43 and 8.47-8.69 ppm for free and hydrogen bonded $\mathrm{NH}$, respectively, and aromatic protons are found in the resonating frequency range 7.09-7.91 ppm (Rauf MK 2008). A comparative ${ }^{1} \mathrm{HNMR}$ spectral study of the complexes (4-15) reveals that the $\mathrm{N}-\mathrm{H}$ signal appears at $12.43,12.39$, and $12.27 \mathrm{ppm}$ in the ${ }^{1} \mathrm{H}$ NMR spectrum of ligands $\mathbf{1}, \mathbf{2}$ and $\mathbf{3}$ respectively, and is not present in the corresponding complexes as a consequence of coordination, in agreement with IR results. Furthermore, all the other chemical shift values of ${ }^{1} \mathrm{H}$ NMR data agree with the literature and expected structure of the complex (Fig 1). Hence, all the characteristic resonating shifts were identified by their intensity and multiplicity patterns (Hakan A 2003; Losada J 2000).

\subsection{Assay for urease inhibition}

Transition metal complexes of thioureas were evaluated against Jack bean urease in vitro. The results are presented in Table 3 . Initially the compounds were screened at a concentration of $1 \mathrm{mM}$. The compounds which exhibited 
more than $50 \%$ inhibition were further selected for complete characterization. All of the transition metal complexes of thioureas were potent inhibitors of Jack bean urease. Thiourea was used as the reference compound for the assay, and its value is also included in Table 3 . The screened compounds exhibited excellent inhibitory activity in micromolar range. Among the tested compounds, $\mathbf{5}$ was the most potent with $\mathrm{IC}_{50}$ being $14.1 \mu \mathrm{M}$. Other potent compounds were 8,11 and 12 having $16.5,18.0$ and $16.9 \mu \mathrm{M}$, respectively. Upon further modification, moderate activity was exhibited by $4,6,9,10,13$ and 14 , their $\mathrm{IC}_{50}$ ranging from 24.6-58.1 $\mu \mathrm{M}$. Among the synthesized analogues, compound 7 was least potent with $\mathrm{IC}_{50}$ values greater than $68.9 \mu \mathrm{M}$. Compound 5 has showed potent inhibition due to presence of chloro group at meta position and metal ligand is Co (II) in this case. The presence of chloro group at meta position enhances the urease inhibition activity as shown by results in case of compounds $\mathbf{5 , 8}$ and 11. Whereas, the substitution of bromo group at same position reduces the activity many folds irrespective of metal ligand attached i-e; compounds 4,7 , 10 and 13 (Table 3).

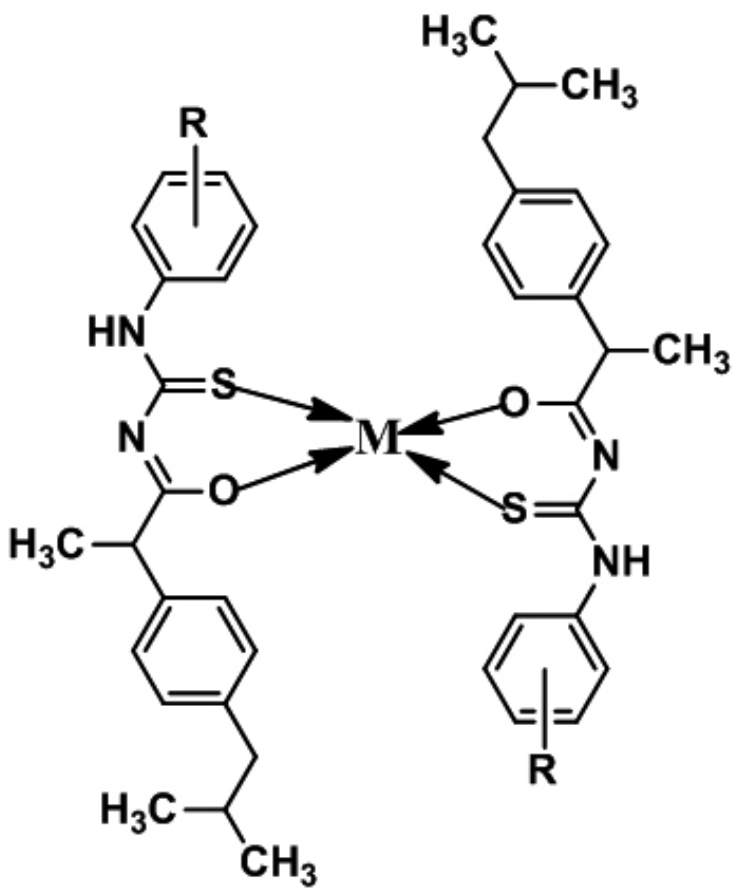

Fig 1: Most expected structure of the metal( $\mathrm{Cu}(\mathrm{II}), \mathrm{Co}(\mathrm{II}), \mathrm{Ni}(\mathrm{II}), \mathrm{Pb}$ (II) ) complexes of ibuprofen substituted thioureas.

Table 3 Inhibitory activity of metal complexes of thioureas (4-15) against Jack bean urease.

\begin{tabular}{|c|c|c|c|}
\hline Compounds & $\mathbf{R}$ & $\mathbf{M X}$ & $\mathbf{I C}_{\mathbf{5 0}}(\boldsymbol{\mu} \mathbf{M}) \pm \mathbf{S E M}$ \\
\hline $\mathbf{4}$ & $2-\mathrm{Br}$ & $\mathrm{Co}$ & $32.9 \pm 14.1$ \\
\hline $\mathbf{5}$ & $2-\mathrm{Cl}$ & $\mathrm{Co}$ & $14.6 \pm 3.3$ \\
\hline $\mathbf{6}$ & 3,4-Dichloro & $\mathrm{Co}$ & $24.6 \pm 7.45$ \\
\hline $\mathbf{7}$ & $2-\mathrm{Br}$ & $\mathrm{Ni}$ & $68.9 \pm 4.92$ \\
\hline $\mathbf{8}$ & 2-Cl & $\mathrm{Ni}$ & $16.5 \pm 10.4$ \\
\hline $\mathbf{9}$ & 3,4-Dichloro & $\mathrm{Ni}$ & $34.9 \pm 4.51$ \\
\hline $\mathbf{1 0}$ & 2-Br & $\mathrm{Pb}$ & $58.1 \pm 18.2$ \\
\hline $\mathbf{1 1}$ & 2-Cl & $\mathrm{Pb}$ & $18.0 \pm 3.94$ \\
\hline $\mathbf{1 2}$ & 3,4-Dichloro & $\mathrm{Pb}$ & $16.9 \pm 8.51$ \\
\hline $\mathbf{1 3}$ & 2-Br & $\mathrm{Cu}$ & $37.3 \pm 25.9$ \\
\hline $\mathbf{1 4}$ & 2-Cl & $\mathrm{Cu}$ & $37.7 \pm 0.76$ \\
\hline $\mathbf{1 5}$ & 3,4-Dichloro & $\mathrm{Cu}$ & \\
\hline Standard & Thiourea & - & $21.1 \pm 1.23$ \\
\hline
\end{tabular}

\section{CONCLUSION}

A series of thiourea derivatives bearing ibuprofen moiety $\mathrm{N}$-2-(4-(2methylpropyl)phenyl)propionoyl- $N^{\prime}-\left(2^{\prime}\right.$-bromophenyl) thiourea (1), $N$-2-(4(2-methylpropyl)phenyl)propionoyl- $N^{\prime}-\left(2^{\prime}\right.$-chlorophenyl) thiourea (2), $N$-2(4-(2-methylpropyl)phenyl)propionoyl- $N^{\prime}-\left(3^{\prime}, 4^{\prime}\right.$-dichlorophenyl) thiourea (3) and their corresponding complexes of $\mathrm{Co}(\mathrm{II})$ (4-6), $\mathrm{Ni}(\mathrm{II})$ (7-9), $\mathrm{Pb}$ (II) $(10-12)$ and $\mathrm{Cu}(\mathrm{I})(13-15)$ were synthesized and characterized by FTIR and ${ }^{1} \mathrm{HNMR}$ spectroscopy. The synthesized thiourea derivatives were evaluated for Jack bean urease inhibition activity. All of the compounds showed significant inhibition against Jack bean urease due to the similarity of their basic skeleton with urease substrate. The compound (5) showed excellent inhibitor activity having $\mathrm{IC}_{50}$ value $14.6 \pm 3.3 \mathrm{M}$.

\section{ACKNOWLEDGEMENT}

A. M greatly thankful to the Higher Education Commission of Pakistan for providing funds under IPFP sheme. J. I is greatful to COMSTECH-TWAS and German-Pakistani Research Collaboration Programme under DAAD project for financial support.

\section{REFERENCES}

1. Amtul Z RA, Siddiqui RA, Choudhary MI (2002) Chemistry and mechanism of urease inhibition. Curr Med Chem 9:1323-1348

2. Arslan H, Flörke U, Külcü N, Emen M (2006) Crystal structure and thermal behaviour of copper (II) and zinc (II) complexes with N-pyrrolidine-N'-(2chloro-benzoyl) thiourea Journal of Coordination Chemistry 59:223-228

3. Aslam MA MS, Shahid M, Saeed A, Iqbal J (2011) Synthesis, biological assay in vitro and molecular docking studies of new Schiff base derivatives as potential urease inhibitors. Eur J Med Chem 46:5473-5479 doi:doi: 10.1016/j.ejmech.2011.09.009.

4. Bacanamwo M WC-P, Lubbers M, Polacco J, (2002) Activation of the urease of Schizosaccharomyces pombe by the UreF accessory protein from soybean. Mol Genet Genomics 268:525-534

5. Benini S (1999) A new proposal for urease mechanism based on the crystal structures of the native and inhibited enzyme from Bacillus pasteurii: why urea hydrolysis costs two nickels. Structure 7:205.

6. Beyer L HE, Hennig H, Kirmse R, Hartmann H, Liebscher J (1975) Synthese and charakterisierung neuartiger übergangsmetallchelate von 1,1-dialkyl-3-benzoyl-thioharnstoffen J Prakt Chem 317:829 - 839

7. Bundy LG BJ (1973) Effects of substituted p-benzoquinones on urease activity in soils. Soil Biol Biochem 5:847-853

8. Carcu V NM, Rosu T, Serban S (2000) Synthesis, Characterization of Complexes of N-benzoyl-n'-2-nitro-4-methoxyphenyl-thiourea with $\mathrm{Cu}, \mathrm{Ni}, \mathrm{Pt}, \mathrm{Pd}, \mathrm{Cd}$ and $\mathrm{Hg}$. J Therm Anal Calorim 61:935-945 doi:1023/A:1010163012703

9. Costa C, Gushikem Y (1984) Interaction of N-acylthiourea nickel (II) chelates with nitrogen bases in solution and in the solid state. Inorganica chimica acta 90:133-136

10. D'Cruz OJ DY, Uckun FM, (2003) Potent dual anti-HIV and spermicidal activities of novel oxovanadium(V) complexes with thiourea nonnucleoside inhibitors of HIV-1 reverse transcriptase Biochemical and biophysical research communications 302:253-264

11. Del CR CJ, García E, Hermosa RR, Jiménez-Sánchez A, Manzano JL, Monte E, Rodríguez-Fernández E., Sanz F (2002) Thiourea derivatives and their nickel(II) and platinum(II) complexes: antifungal activity. J Inorg Biochem 89:74-82 doi:http://dx.doi.org/10.1016/S0162-0134(01)00408-1

12. Dhumane NR HS, Nawarkhele VV, Shirsat MD, (2006) Dielectric studies of metal complexes of thiourea crystals for electro $\square$ optic modulation. Cryst Res Technol 41:897-901

13. Douglass IB, Dains FB (1934) Some derivatives of benzoyl and furoyl isothiocyanates and their use in synthesizing heterocyclic compounds. J Am Chem Soc 56:719-721

14. Gripenberg-Lerche C ZL, Ahtonen P, Toivanen P, Skurnik M (2000) Construction of urease-negative mutants of yersinia enterocolitica serotypes o: 3 and o: 8: role of urease in virulence and arthritogenicity. Infect Immun 68:942-947.

15. Hakan A NK, Ulrich F (2003) Synthesis and characterization of copper(II), nickel(II) and cobalt(II) complexes with novel thiourea derivatives. Transition Met Chem 287:816-819

16. Hanif M SK, Saleem M, Rama NH, Zaib S, Iqbal J (2012) Synthesis, urease inhibition, antioxidant, antibacterial, and molecular docking studies 
of 1, 3, 4-oxadiazole derivatives. ISRN pharmacology 2012 doi:doi. org/10.5402/2012/928901

17. Holm LS, Chris, (1997) Proteins Struct Funct Genet 28:72-82.

18. KD T (2003) Drugs for peptic ulcer. In: In Essentials of Medical Pharmacology. 5th edn. Jaypee Brothers Medical Publishers, New Delhi, pp 587-598

19. Kobashi K HJ, Uehara K (1962,) Specific inhibition of urease by hydroxamic acids. Biochim Biophys Acta 65:380.

20. Kot M ZW, Orlinska K (2001) Inhibition of jack bean urease by N-(nbutyl) thiophosphorictriamide and $\mathrm{N}$-(n-butyl) phosphorictriamide: determination of the inhibition mechanism J Enzyme Inhib 16:507-516

21. Krajewska B (2009) Ureases I. Functional, catalytic and kinetic properties: A review. J Mol Catal B Enzym 59:9-21

22. Kuehler TC FJ, Bergman NA, Weilitz J, Lee A, Larsson H (1995) Structure-activity relationship of omeprazole and analogs as Helicobacter pylori urease inhibitors J Med Chem and Res 38:4906-4916

23. Kumavat P, Jangale AD, Patil DR, Dalal KS, Meshram JS, Dalal DS (2013) Green synthesis of symmetrical N, N'-disubstituted thiourea derivatives in water using solar energy. Environ Chem Lett 11:177-182

24. Kumavat PP JA, Patil DR, Dalal KS, Meshram JS, Dalal DS (2013) Green synthesis of symmetrical $\mathrm{N}, \mathrm{N}^{\prime}$-disubstituted thiourea derivatives in water using solar energy. Environ Chem Lett 11:177-182

25. Losada J PI, Beyer L (2000) Synthesis, electrochemical properties and electro-oxidative polymerizationof copper(II) and nickel(II) complexes with N'-benzoylthiourea ligands containing pyrrole. Transition Met Chem 25:112-117

26. McMillan DC LE, Smith J, Sturgeon C, Preston T, Cooke TG, McArdle CS, (1995) Effect of extended ibuprofen administration on the acute phase protein response in colorectal cancer patients. Eur J Surg Oncol 21:531534

27. Mobley H, Island MD, Hausinger RP (1995) Molecular biology of microbial ureases Microbiological reviews 59:451-480

28. Mobley HL HR (1989) Microbial ureases: significance, regulation, and molecular characterization Microbiol Rev 53:85-108

29. Moro AC MA, Netto AV, Ananias SR, Quilles MB, Carlos IZ, Pavan FR, Leite CQ, Hoer M (2009) Antitumor and antimycobacterial activities of cyclopalladated complexes: X-ray structure of $[\mathrm{Pd}(\mathrm{C} 2, \mathrm{~N}-\mathrm{dmba})(\mathrm{Br})$ (tu)] $(\mathrm{dmba}=\mathrm{N}, \mathrm{N}$-dimethylbenzylamine, tu=thiourea). Eur J Med Chem 44:4611-4615

30. Muri EMF MH, Avery MA Williamson JS (2003) Design and Synthesis of Heterocyclic Hydroxamic Acid Derivatives as Inhibitors of Helicobacter pylori Urease. Synthetic Commun 33:1977

31. Nadeem S RM, Ahmad S, Ebihara M, Tirmizi SA, Bashir SA, Badshah A (2009) Synthesis and characterization of palladium (II) complexes of thioureas. X-ray structures of [Pd (N, N'-dimethylthiourea) 4] $\mathrm{Cl} 2 \cdot 2 \mathrm{H} 2 \mathrm{O}$ and [Pd (tetramethylthiourea) 4] Cl2 Transition Met Chem 34:197-202

32. Nagata K SH, Iwahi T, Shimoyama T, Tamura T (1993) Potent inhibitory action of the gastric proton pump inhibitor lansoprazole against urease activity of helicobacter pylori: unique action selective for $\mathrm{H}$. pylori cells. Antimicrob Agents Chemother 37:769-774

33. Rao P Kea (2008) Evolution of nonsteroidal anti-inflammatory drugs (nsaids): Cyclooxygenase (cox) inhibition and beyond. J Pharm Pharm 11:81-110

34. Rasmussen CR VFJ, Weaner, Reynolds BE, Hood AR, Hecker LR, Nortey SO, Hanslin A, Costanzo MJ, Powell ET, Molinari AJ (1988) Improved procedures for the preparation of cycloalkyl, arylalkyl and arylthioureas. Synthesis 6:456-459 doi:10.1055/s-1988-27605

35. Rauf MK BA, Bolte M, Imtiaz-ud-Din (2006) 1-(2,6-Dichlorobenzoyl)3-(2-chlorophenyl) thiourea toluene hemi solvate. Acta Crystallogr E62:03553.

36. Rauf MK BA, Bolte M, Imtiaz-ud-Din (2010) Solution-phase synthesis of a combinatorial n, n'-disubstituted thioyrea library: Complexation, characterization and biological studiest. Quaid-i-Azam University, Islamabad

37. Rauf MK BA, Gielen M, Ebihara M, Vos D, Ahmed S (2009) Synthesis, structural characterization and in vitro cytotoxicity and anti-bacteria activity of some copper(I) complexes with N,N'-disubstituted thioureas. J Inorg Biochem 103:1135-1144 doi:10.1016/j.jinorgbio.2009.05.014

38. Rauf MK SA, Bolte M, Badshah A, Mirza B, (2008) Synthesis, characterization and biological activities of some new organotin(IV) derivatives: Crystal structure of $[(\mathrm{Sn} \mathrm{Ph} 3)(\mathrm{OOCC} 6 \mathrm{H} 4 \mathrm{OH})]$ and [(SnMe3)2 (OOC)2C6Cl4 (DMSO)2]. J Organomet Chem 693:3043-3048
39. Saeed A, Khan MS, Rafique H, Shahid M, Iqbal J (2014) Design, synthesis, molecular docking studies and in vitro screening of ethy 4-(3-benzoylthioureido) benzoates as urease inhibitors. Bioorganic chemistry $52: 1-7$

40. Saeed A, Zaib S, Pervez A, Mumtaz A, Shahid M, Iqbal J (2013) Synthesis, molecular docking studies, and in vitro screening of sulfanilamidethiourea hybrids as antimicrobial and urease inhibitors J Med Chem and Res 22:3653-3662

41. Sun MZ WF, Wu YM, Liu WM (2008) A ditopic colorimetric sensor for fluoride ion based on thiourea mercury complex. Spectrochim Acta 71:814-817

42. Todd MJ HR (1989) Competitive inhibitors of Klebsiella aerogenes urease. Mechanisms of interaction with the nickel active site J Biol Chem 264:15835-15842

43. Tremblay L DG, Ghali E, McMullen J, Lanouette M, (1996) Gold recovery from a sulfide bearing gold ore by percolation leaching with thiourea. Int J Miner Process 48:225-244

44. Weatherburn M (1967) Phenol-hypochlorite reaction for determination of ammonia Analytical chemistry 39:971-974

45. Yuan YF WJ, Gimeno MC, Laguna A., Jones PG, (2001) Synthesis and characterisation of copper complexes with N-ferrocenoyl-N "(alkyl) thioureas Inorg Chimica Acta 324:309-317 